\title{
In-Situ Dissolution Testing Using Different UV Fiber Optic Probes and Instruments
}

\author{
Xujin Lu ${ }^{1,2}$, Ruben Lozano ${ }^{2}$, Pankaj Shah ${ }^{2}$
}

Email:xujin.lu@bms.com

\begin{abstract}
:
In-situ dissolution testing using UV fiber optics has attracted much attention in the pharmaceutical industry. Several instruments with different designs of fiber optic probes have been applied for this testing. These instruments employ different types of spectrometers and the probes differ in shape, light beam path, and sampling window. These differences may have significant impact on the dissolution operations and results. In this article we will examine these instruments and probes and discuss the effects of their characteristics on dissolution testing.
\end{abstract}

\section{Introduction:}

Fiber optic technology for dissolution testing has been a topic of interest for many researchers for some years. Josefson, Johansson, and Tortensson ${ }^{1}$ published early research in this field in 1988. They explored the feasibility of using UV fiber optics for in-situ dissolution and to overcome sample turbidity interference without filtration. In 1993, Brown and $\mathrm{Lin}^{2}$ used a single optical fiber and a photo diode array (PDA) UV/vis spectrometer to track dissolution in a single vessel. Their work was extended thereafter to use six optical fibers and a PDA spectrometer for multiple dissolution vessels ${ }^{3}$. In 1995, Cho and coworkers developed a sevenchannel fiber optic dissolution system using a spectrometer with two-dimensional chargecoupled devices (CCD), which allowed the simultaneous monitoring of six dissolution vessels and a seventh reference vessel ${ }^{4,5}$. Several other studies around the same time further explored the possibility of using fiber optic technology for special dissolution applications. For example, Chen and his group ${ }^{6,7}$ employed a fiber optic based chemical sensor for continuous monitoring of in-situ dissolution. Gemperline et al ${ }^{8}$ applied a CCD/fiber optic system for determining two-component dissolution profiles of a pharmaceutical product, which used a full-range spectral principal component regression methodology. Aldridge and coworkers ${ }^{9}$ automated a single-probe fiber optic system that used a robot arm to move the probe from vessel to vessel for sequential testing. All these breakthrough studies had great impact on subsequent development of commercial instruments and established the platform for modern in-situ fiber optic dissolution testing.

Commercial UV fiber optic dissolution instruments became available in 1999. Several manufac- turers in the United States introduced their independently developed instruments one after another. These commercial instruments employed either a spectrometer with multi-channel CCDs ${ }^{10}$, ${ }^{11}$, multiple PDA spectrometers ${ }^{12,13}$, or a scanning spectrometer with a mechanical multiplexer ${ }^{14,15}$. Commercialization of this technology and its consequent availability to a broad range of users has attracted much attention from the pharmaceutical industry. Recent publications and research works further described the capability of this technology ${ }^{16-18}$ and demonstrated that UV fiber optics is a breakthrough for dissolution testing. A regulatory perspective on this technology has also been discussed in a recent article ${ }^{19}$.

\section{Why Fiber Optic Dissolution?}

Traditionally, dissolution testing has been conducted by manually or automatically removing samples from dissolution vessels, and then bringing the samples either to a UV spectrometer for determination of the analyte concentration, or to a HPLC for UV or fluorescence detection after separation. The use of fiber optics changes this traditional way of sampling. Instead of bringing the samples to a UV spectrometer, fiber optics brings the UV spectrometer to the sample solutions. A real-time drug release level is determined in-situ or in the vessels without sample removal.

In-situ dissolution using fiber optics has significant advantages over traditional dissolution. The labor-intensive manual sampling procedure is eliminated and the testing procedure is simplified. It is more economical because consumables for sampling, such as pipettes, syringes, sippers, tubing, and filters, are not needed. Without sampling, it creates a new way for automation consideration. In addition, fiber optics can generate more frequent
${ }^{1}$ Corresponding author, Senior Research Investigator, Pharmaceutical Research Institute, Bristol-Myers Squibb Company, Analytical R\&D, One Squibb Drive,New Brunswick, NJ 08903,email:xujin.lu@bms.com
2Pharmaceutical Research Institute, Bristol-Myers Squibb Company, Analytical $R \& D$, New Brunswick, NJ. 
data points and produce a more detailed dissolution profile, which may be very beneficial for showing a method's discriminating ability.

\section{Challenges of Fiber Optic Dissolution}

Although fiber optic dissolution has advantages, it also faces a number of challenges. First, dissolution is a restrictively regulated test. It takes time for scientists, managers, and regulatory agencies in the pharmaceutical industry to accept replacement of current methodology with a new technique. Second, fiber optics is a cutting edge technology with a relatively short history of dissolution applications. The instrumentation is not yet mature, and optimization of the hardware and software is still in progress. Furthermore, to most users, the different spectroscopic designs among the commercial instruments complicate its selection and implementation. For example, an instrument with a conventional scanning UV spectrometer may detect dissolution solutions in six or twelve vessels in a sequential mode, whereas an instrument with PDA or CCD devices may detect these solutions simultaneously. Users have to understand these different characteristics and their impact in order to develop a fiber optic method and conduct a successful dissolution test.

In addition to different spectroscopic designs, the UV fiber optic probes provided with different instruments add more complexity to the applications. The UV fiber optic probe is placed in a dissolution vessel for in-situ concentration determination. Based on the shape and the location where they are placed in the vessel, probes can be categorized into three types (see Figure 1): (1) Shaft probe, which is fixed in a hollow shaft and placed at the center of the

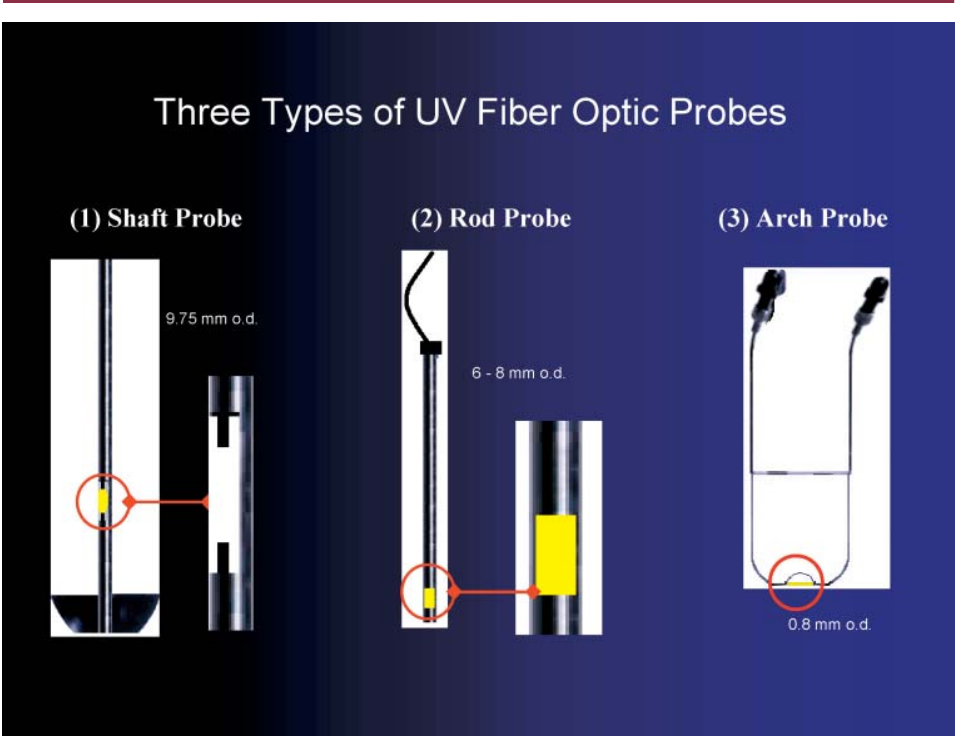

Figure 1. Three types of UV fiber optic probes. vessel. (2) Rod probe, essentially a solid rod dipped in the vessel, with a detection window in the traditional sampling location defined by USP. There are two types of rod probe made by different vendors with slightly different sizes and with a built-in mirror or lens. (3) Arch probe, which has an arch shape and a detection window in the traditional USP sampling location. These probe types have different dimensions, light beam paths, and sampling windows, which may significantly affect the dissolution testing. These effects maybe due to changes in hydrodynamics, stray light, light scattering, particulate accumulation, etc.

The purpose of this study was to examine different types of UV fiber optic instrumentation and to evaluate the effects of their spectroscopic characteristics on dissolution testing. Experimental results and pharmaceutical application examples will be discussed to demonstrate the characteristics of the technology and the limitations of the instrumentation.

\section{Experimental and Materials Instrumentation}

Three UV fiber optic instruments were used in this study (Figure 2). They were:(1) Opt-Diss Fiber Optic UV system (Leap Technologies Inc.) with a multi-channel CCD spectrometer $(205-410 \mathrm{~nm})$, one set of six shaft probes (pathlength $1 \mathrm{~cm}$ ), two sets of six arch probes (pathlengths of 1 and $10 \mathrm{~mm}$ ), and a Hanson SR8 dissolution bath. (2) Rainbow Dynamic Monitor (Delphian Technology LP) with six PDA detectors (230 - $400 \mathrm{~nm}$ ), six Delphian rod probes equipped with two sets of probe tips (pathlengths of 2 and $10 \mathrm{~mm}$ ), and a Vankel 7010 bath with a manifold to fix and raise the probes. (3) IO Fiber Optic Dissolution system (C Technologies) with a Varian Cary 50 scanning spectrometer (190 - $1100 \mathrm{~nm}$ ), a Cassini mechanical multiplexer, six C-Tech rod probes (pathlength of $10 \mathrm{~mm}$ ), and a Vankel 7025 bath with a manually staggered drop function for baskets.

\section{Hydrodynamic Effect Study}

The arch probes were tested on USP dissolution apparatus 1 and 2 , and C Tech rod probes were tested on USP apparatus 2. The USP disintegrating calibrator, $10-\mathrm{mg}$ Prednisone tablet (Lot M) and USP reference standard Prednisone (Lot $\mathrm{L}$ ) were used. The vessels were stirred at $100 \mathrm{rpm}$ for apparatus 1 and at $50 \mathrm{rpm}$ for apparatus 2. The dissolution medium preparation and manual testing procedure were followed as per the USP method ${ }^{20}$.

For each apparatus, two runs were performed on a total of 12 tablets. During the first run, three vessels (\#4-6) contained the arch or rod fiber optic probes and three vessels (\#1-3) were without probes. All six vessels were manually sampled at 30 minutes, and these sample solutions were detected offline (using a Perkin Elmer Lambda 20 UV spectrometer). 


\section{In-Situ Dissolution Testing Using Different ... continued}

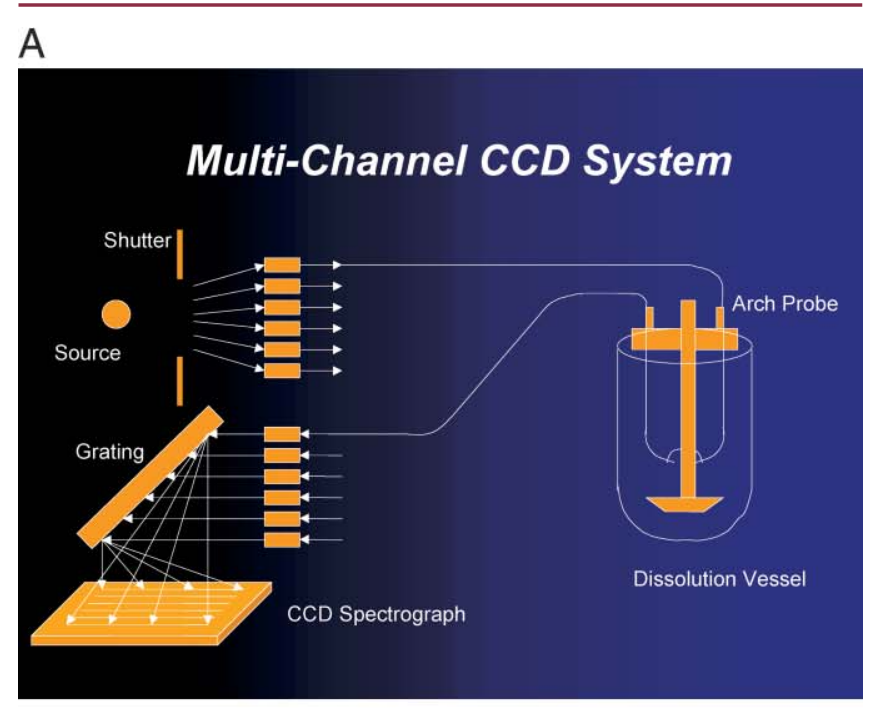

B

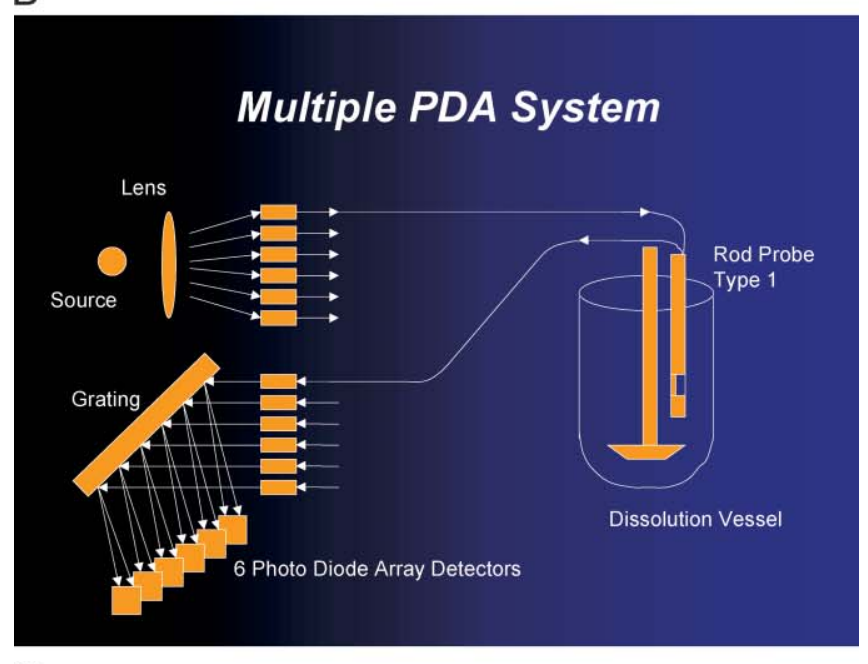

C

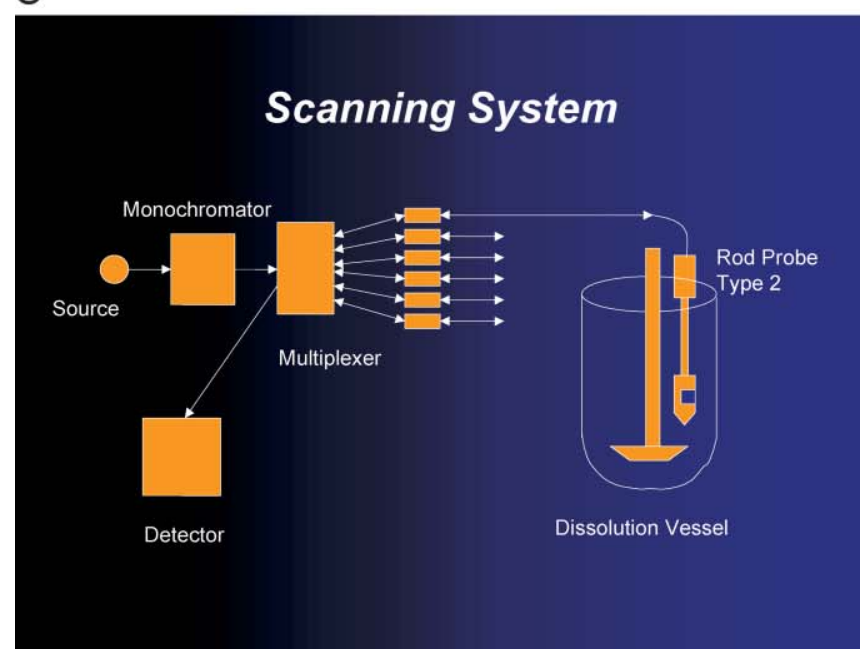

Figure 2. Schematics of the three types of fiber optic dissolution systems. (A) Multi-Channel CCD System; (B) Multiple PDA System; (3) Scanning System.
Table 1. Dissolution Test Conditions for an ExtendedRelease Tablet Formulation.

\begin{tabular}{|ll|}
\hline Parameter & Description \\
\hline Media & $\begin{array}{l}0.05 \mathrm{M} \text { Phosphate Buffer, } \mathrm{pH} 6.8, \\
\text { with 0.006\% EDTA, 1000 } \mathrm{mL}, 37.0^{\circ} \mathrm{C}\end{array}$ \\
\hline Apparatus & Basket, $100 \mathrm{rpm}$ \\
\hline Run Time & 16 hours \\
\hline \multirow{2}{*}{ Fiber Optic } & Arch probe / Opt-Diss system \\
Method & $\begin{array}{l}\text { Baseline correction: } 350 \mathrm{~nm} \\
\text { Pathlength: } 10 \mathrm{~mm}\end{array}$ \\
\hline Manual & Manual sampling followed by HPLC analysis \\
Method & UV detection: $210 \mathrm{~nm}$. \\
\hline
\end{tabular}

Simultaneously, the three vessels with the probes were also detected using the UV fiber optic system. The positions were switched for the second run; the probes were placed into vessels \#1-3, while vessels \#4-6 were without probes. The manual sampling/offline UV detection and fiber optic in-situ detection was then repeated.

\section{Linear Range Study}

A serial dilution $(0.005-0.25 \mathrm{mg} / \mathrm{mL})$ of potassium chromate (Baker Analyzed ACS Reagent, purchased from J.T. Baker, Lot N42618) solution in $5 \mathrm{mM}$ sulfuric acid (GR, purchased from EM Science, Lot 36149) was used for this study. The IO system with C Tech 1-cm rod probes and the Opt-Diss system with 1-cm arch probes were tested.

Shutter exposure time for the $1-\mathrm{cm}$ arch probes was 750 $\mathrm{ms}$. The spectral resolution was $1 \mathrm{~nm}$. The average absorption measurement at $257 \mathrm{~nm}$ from six probes was calculated for each data point.

\section{Light Scattering Effect}

An immediate-release 50-mg capsule formulation of a BMS drug was tested on the Opt-Diss system with 1-cm shaft probes and the Rainbow system with Delphian $1-\mathrm{cm}$ rod probes. The dissolution conditions are: $1000 \mathrm{~mL}$ of 0.05 $\mathrm{M}$ phosphate buffer at $\mathrm{pH} 6.8$, USP apparatus 2 at $50 \mathrm{rpm}$.

\section{Particulate Accumulation Effect}

Table 1 shows the dissolution method and conditions used for the testing of an extended-release 10-mg tablet formulation from BMS drug development.

\section{Results and Discussion \\ Hydrodynamic Effect}

"Hydrodynamic effect" refers to an interruption of the normal hydrodynamic flow within the vessel that may 
Table 2. Hydrodynamic Effect Test for Arch Probes with USP Apparatus 1.

\begin{tabular}{|c|c|c|c|c|c|c|}
\hline \multicolumn{7}{|c|}{ Dissolution Results for 10-mg Prednisone Calibrator (30 $\mathrm{min}$ )" } \\
\hline & \multicolumn{2}{|c|}{ No Probe } & \multicolumn{2}{|c|}{ Arch Probe in Place } & \multicolumn{2}{|c|}{ Arch Probe in Place } \\
\hline & Manual & (Vessel \#) & Manual & (Vessel \#) & Fiber Optic & (Vessel \#) \\
\hline \multirow{3}{*}{ Run-1 } & 74.9 & $(1)$ & 72.7 & (4) & 72.9 & (4) \\
\hline & 69.8 & $(2)$ & 66.0 & $(5)$ & 66.4 & $(5)$ \\
\hline & 74.5 & (3) & 73.6 & (6) & 72.5 & (6) \\
\hline \multirow{3}{*}{ Run-2 } & 68.9 & (4) & 70.8 & (1) & 71.1 & (1) \\
\hline & 67.1 & (5) & 72.5 & (2) & 70.2 & (2) \\
\hline & 72.2 & (6) & 69.9 & (3) & 71.6 & (3) \\
\hline Average & 71.2 & & 70.9 & & 70.8 & \\
\hline SD & 3.15 & & 2.76 & & 2.36 & \\
\hline Difference & vs Arch/Manual & 0.3 & vs Arch/Fiber Optic & 0.1 & vs Manual & -0.4 \\
\hline
\end{tabular}

Table 3. Hydrodynamic Effect Test for Arch Probes with USP Apparatus 2.

\begin{tabular}{|lcccccc|}
\hline \multicolumn{7}{c}{ Dissolution Results for 10-mg Prednisone Calibrator (30 min) } \\
\hline & \multicolumn{2}{c}{ No Probe } & Arch Probe in Place & \multicolumn{2}{c|}{ Arch Probe in place } \\
\cline { 2 - 7 } Run-1 & Manual & (Vessel \#) & Manual & (Vessel \#) & Fiber Optic & (Vessel \#) \\
\cline { 2 - 8 } & 28.2 & $(1)$ & 31.2 & $(4)$ & 30.6 & $(4)$ \\
\hline & 28.7 & $(2)$ & 29.1 & $(5)$ & 28.1 & $(5)$ \\
\hline & 34.7 & $(3)$ & 30.7 & $(6)$ & 29.4 & $(6)$ \\
\hline Run-2 & 30.0 & $(4)$ & 29.8 & $(1)$ & 29.4 & $(1)$ \\
\hline Average & 29.6 & $(5)$ & 29.4 & $(2)$ & 29.4 & $(2)$ \\
\hline SD & 31.7 & $(6)$ & 30.0 & $(3)$ & 29.5 & $(3)$ \\
\hline Difference & 30.2 & & 30.0 & & 29.4 & \\
\hline
\end{tabular}

result when something, such as a fiber optic probe, is introduced into the medium during a dissolution test. The prednisone tablet was chosen for this test because it requires $500 \mathrm{~mL}$ of dissolution medium, and smaller volumes are more sensitive to such interruption. The extent of the interruption depends on the dimensions of the probe and its location in the vessel, which differs among the three types of UV fiber optic probes.

The arch probe is placed at the sampling position specified by the USP ${ }^{21}$. Based on the USP definition, this location is not less than $1 \mathrm{~cm}$ from the vessel wall and in the middle between the surface of the medium and the top of the rotating basket (in Apparatus 1) or the top of the shaft blade (in Apparatus 2). The outer diameter of an arch probe is $0.8 \mathrm{~mm}$. Its displacement volume is less than 0.15 $\mathrm{mL}$, which was determined to have an insignificant impact on hydrodynamics in the vessel. All results obtained using the arch probes were within the USP acceptance limits of 23-42\% for Apparatus 2 and 64-91\% for Apparatus 1 (See Tables 2 and 3). The difference between vessels with and without probes when using manual sampling was $0.2-0.3$ $\%$ dissolved. The difference between manual 


\section{In-Situ Dissolution Testing Using Different ... continued}

Table 4. Hydrodynamic Effect Test for Rod Probes (C Technologies) with USP Apparatus

\begin{tabular}{|c|c|c|c|c|c|c|}
\hline \multicolumn{7}{|c|}{ Dissolution Results for 10-mg Prednisone Calibrator (30 min) } \\
\hline & No Probe & & Rod Probe in Place & & Rod Probe in Place & \\
\hline & Manual & (Vessel \#) & Manual & (Vessel \#) & Fiber Optic & (Vessel \#) \\
\hline \multirow{3}{*}{ Run-1 } & 30.3 & (1) & 33.9 & (4) & 33.3 & (4) \\
\hline & 28.2 & (2) & 32.9 & (5) & 34.1 & (5) \\
\hline & 29.4 & (3) & 34.4 & (6) & 35.8 & (6) \\
\hline \multirow{3}{*}{ Run-2 } & 29.7 & (4) & 32.1 & (1) & 35.4 & (1) \\
\hline & 28.6 & (5) & 29.6 & (2) & 30.3 & (2) \\
\hline & 27.0 & (6) & 32.4 & (3) & 33.4 & (3) \\
\hline Average & 29.2 & & 32.6 & & 33.8 & \\
\hline SD & 0.84 & & 1.89 & & 2.19 & \\
\hline Difference & vs Rod/Manual & -3.3 & vs Rod/Fiber Optic & -1.2 & vs Manual & $4.6 a$ \\
\hline
\end{tabular}

sampling/offline UV detection and fiber optic in-situ detection using arch probes was $0.4-0.8 \%$ dissolved. Both were not significant to the acceptance limits.

The rod probe is also placed at the USP sampling position. However, the outer diameter of a rod probe is $6 \mathrm{~mm}$ with a thicker, 8-mm head, so the dimensions of a rod probe are significantly larger than the arch probe. At its sampling position, the rod probe may potentially interfere with the hydrodynamic flow in the vessel, usually resulting in faster disintegration and dissolution. This was observed in our study using the C-Tech rod probes (Table 4). Dissolution results for USP prednisone tablets at 30 minutes, using manual sampling with offline UV detection, were about 3\% higher when rod probes were inserted in the media within the vessels, even though all the results were within the USP acceptance range. There was only about $1 \%$ difference between results obtained using fiber optic in situ detection (IO system) versus manual sampling/offline UV detection. Similar results have been reported by Palermo ${ }^{22}$ where $4 \%$ higher dissolution results (absolute) were found for USP calibrators when the Delphian rod probes were used compared to manual sampling.

The shaft probe is located in the center of the dissolution vessel, where it is equivalent to the sampling position in a Sotax dissolution tester. Because no additional object is placed in the vessel, the shaft probe does not generate a hydrodynamic effect. Schatz and coworkers ${ }^{23}$ demonstrated that there is no significant difference in dissolution results between in-situ fiber optic detection with shaft probes and offline UV measurement with manual sampling.

\section{Linear Range}

A broad linear range is desired for any fiber optic dissolution system. As fiber optic dissolution testing is in-situ detection, which does not permit sample dilution, a system with a broad linear range could be very beneficial for testing various dosage levels of pharmaceutical solid formulations. The linear range of a fiber optic based spectroscopic system can be affected by many factors. For example, stray light from the probes ${ }^{24}$, stray light in the spectrometer, detector and electronic noise, system light throughput, and detector dynamic range all may influence the instrument's linear range. When the spectrometer scanning, PDA or CCD - is combined with the fiber optic probes, the resulting linear range of the whole system becomes an important factor in dissolution testing.

For the IO with rod probes and the Opt-Diss with arch probes, the linear ranges are displayed in Figure 3. For 1$\mathrm{cm}$ pathlength, these two systems demonstrated a similar linear range of $0-2.16 \mathrm{AU}$ with correlation coefficients of 0.99966 and 0.99971 , respectively. This was a little surprising because we had expected the IO system to have a higher linear range with the use of the scanning spectrometer. In addition, the C-Tech rod probe has a built-in lens, which may capture stray light but could greatly enhance the window light throughput. The arch probe, in contrast, has no optics and is less efficient at maintaining light throughput at the probe window. Our previous comparisons of these two types of probes on the IO system showed the rod probe has significantly higher transmittance $(25.5 \%$ at $257 \mathrm{~nm})$ than the arch probe $(0.37 \% \text { at } 257 \mathrm{~nm})^{25}$. All these factors should have resulted 


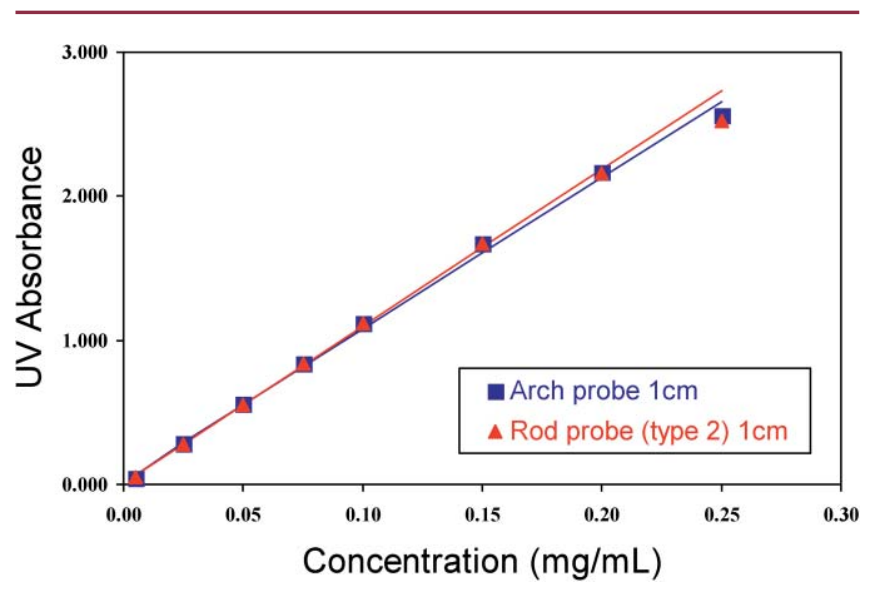

Figure 3. Linearity ranges of rod probes on an 10 system and arch probes on an Opt-Diss system.

in a higher linear range for the rod probe/IO system. However, careful review of the experimental parameters revealed that the Opt-Diss compensated for the low light throughput of the arch probe by increasing shutter exposure time during optimization of the CCD detection condition. In this case, the system linear range is limited not so much by throughput and stray light, but by the dynamic range of the CCD detector, which makes it comparable to the linear range of the IO system.

In comparison with the two systems tested above, Rainbow Dynamic Monitor with Delphian 1.0-cm rod probes was reported to have a linear range of $0-1.5 \mathrm{AU}^{26}$. The result was obtained using the same testing condition and had a linearity correlation coefficient criterion of 0.999 . For the $1.0-\mathrm{cm}$ shaft probes, the linear range measured using Opt-Diss using photometrics ${ }^{27}$ is 0 - 1.8 AU with a 0.999 correlation coefficient.

\section{Light Scattering Effect}

Light scattering effect on fiber optic dissolution testing may come from bubbles, excipients, or undissolved particles passing through or trapped at the probe detection window. Because the fiber optic measurements are performed in-situ without sample filtration, they are susceptible to these interferences. Presence of air bubbles may be due to non-degassed medium, regassing of the medium during the dissolution test, or air trapped at the probe window when the probe is introduced into the medium. Based on the probe geometries, the probability of trapping bubbles is greatest with shaft probes, followed by rod probes, and least with arch probes. A resulting bubble may distort the UV spectra, which may not be able to be rectified by general baseline correction, thus producing incorrect dissolution data.

Figure $4(A-D)$ shows an example of air bubbles affecting spectra measured with six rod probes during a dissolution test of an immediate-release capsule formula-
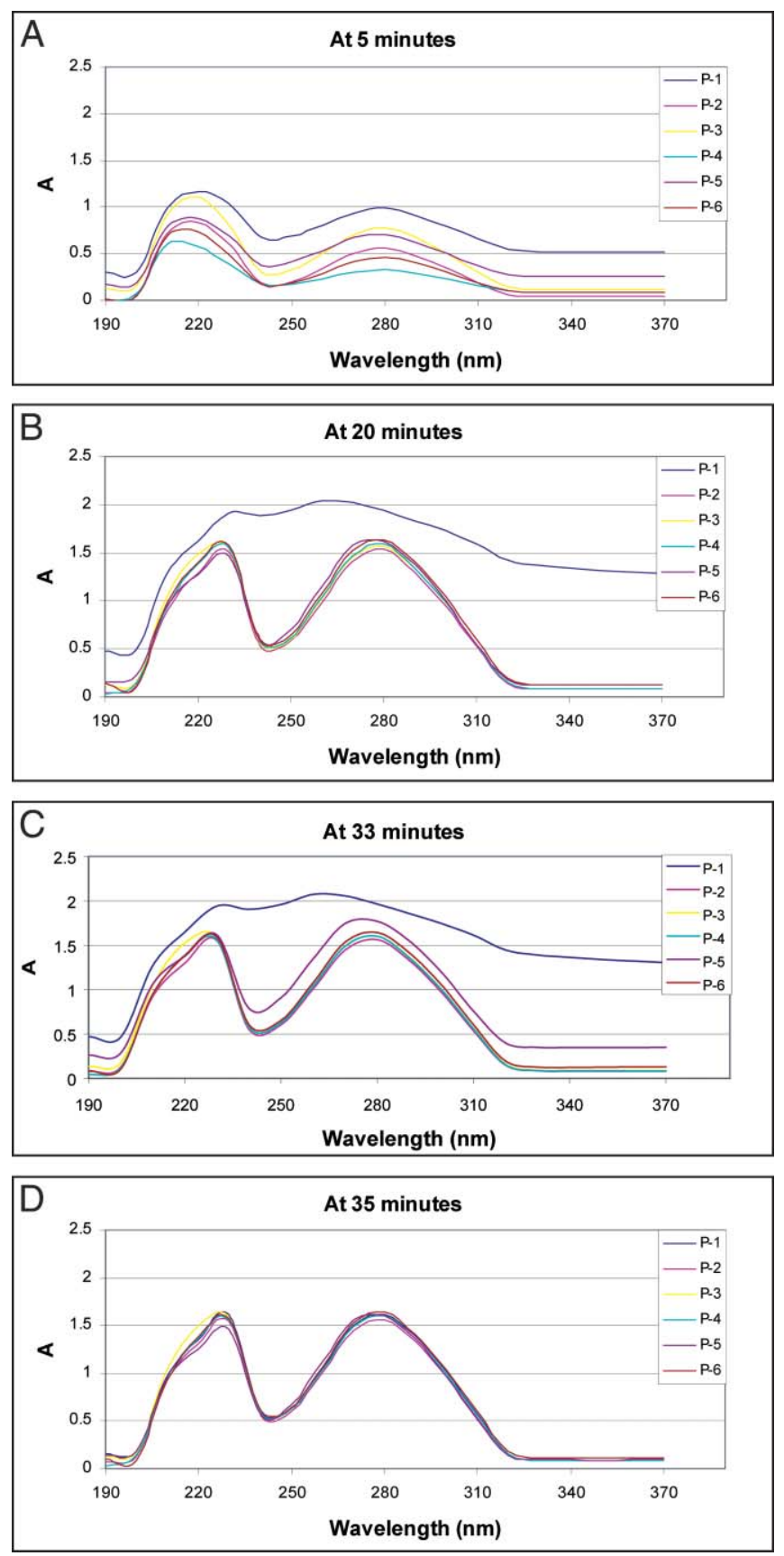

Figure 4. Effect of air bubbles on spectra measured by rod probes during a dissolution test of a capsule formulation. Spectra are shown for 5-min (4A), 20-min (4B), 33-min (4C) and 35-min (4D) time points.

tion. Normal spectra can be observed at the 5 -minute time point. A bubble was then seen to form at the detection window of Probe1, resulting in the spectra shown at 20 and 33 minutes. The bubble was dispersed from the probe window prior to measurement of the 35-minute spectra. In this instance, the bubble distorted (caused higher absorbance) the measurement at the high wavelength $(\sim 350 \mathrm{~nm})$ being used for baseline correction, resulting in 


\section{In-Situ Dissolution Testing Using Different ... continued}

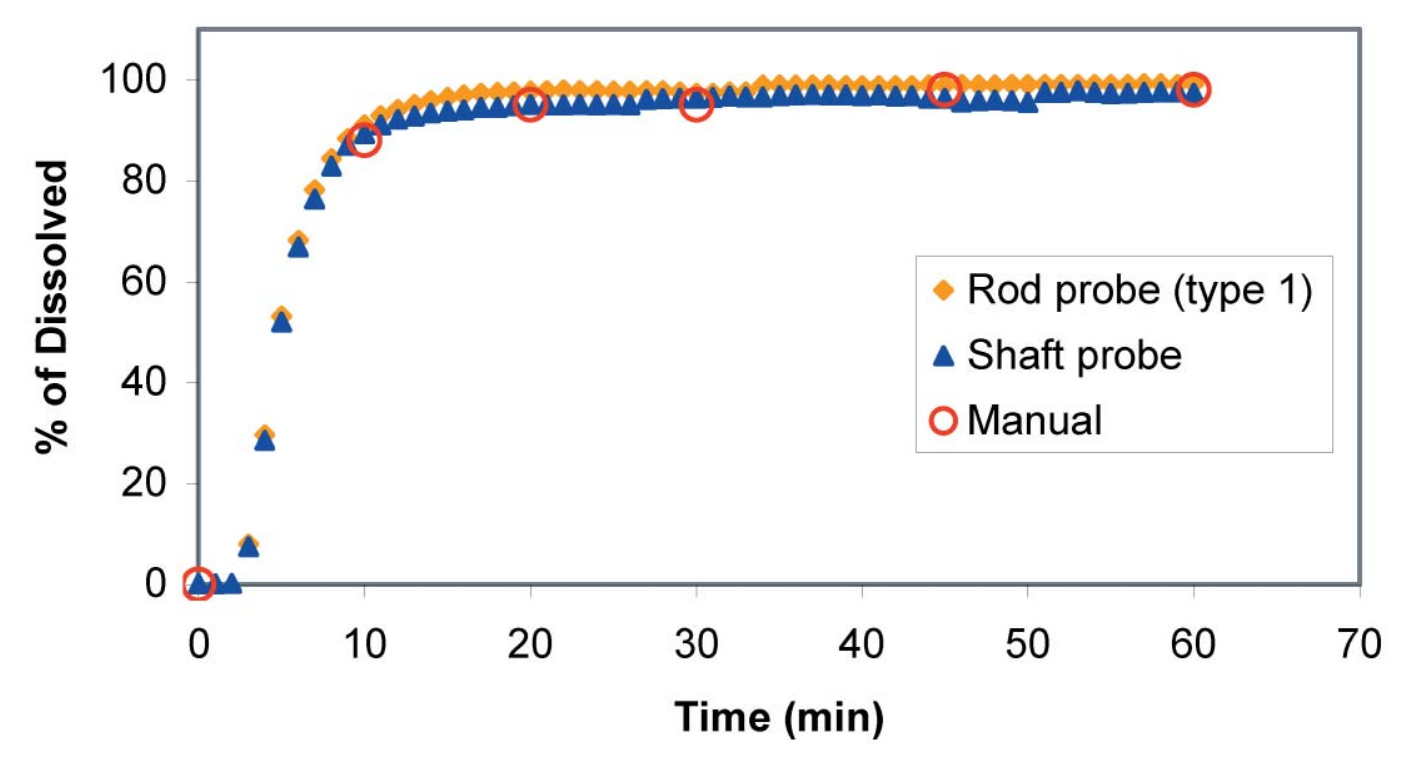

Figure 5. Dissolution profiles of an immediate-release capsule formulation comparing use of shaft probes and rod probes with use of manual sampling and HPLC finish. UV detection: $280 \mathrm{~nm}$ for all the tests. Single-wavelength correction: $350 \mathrm{~nm}$ for the rod probe test. Correction based on a range: 325 - $400 \mathrm{~nm}$ for the shaft probe test.

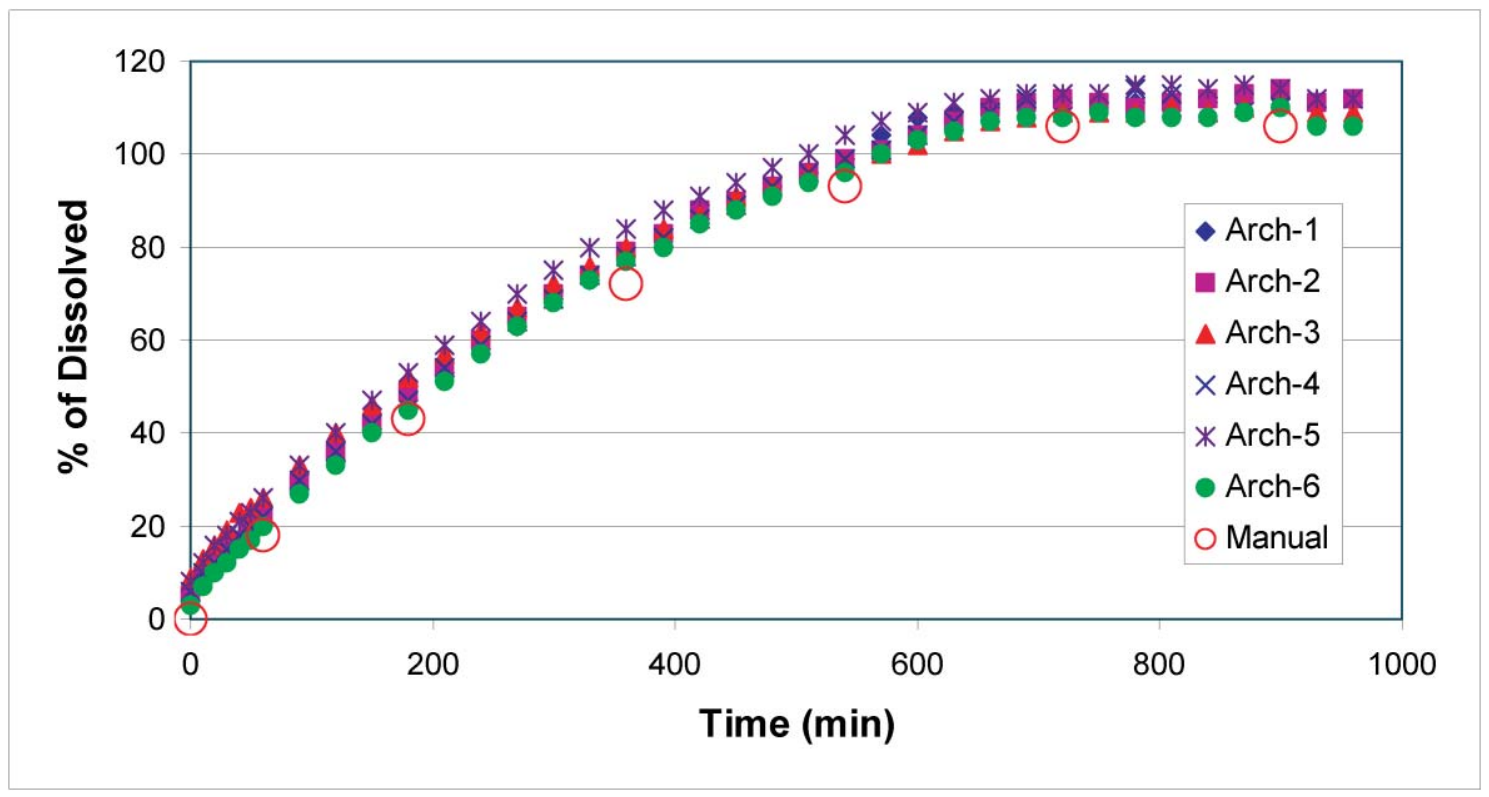

Figure 6. Dissolution profiles of an extended-release 20-mg tablet formulation obtained using the arch rod probe/Opt-Diss versus a manual method. (Sample batch used for the manual testing was different from the batch used for the fiber optic testing). 
inaccurately low dissolution values for Probe1 at the 20and 33-minute time points. Our experience shows it is very important to use welldegassed media for fiber optic dissolution methods. In addition, the probe windows should be carefully checked for bubbles by visual observation before starting a test.

Scattering generated by undissolved particles and excipients can contribute significant interference. This type of scattering may be wavelength-independent or wavelength-dependent. Wavelength-independent scattering is observed more often and is usually manifested by a baseline offset over the entire UV range. This effect can be mediated using single-wavelength correction, two-point correction, or correction based on a range of wavelengths. For most cases, single-wavelength correction is sufficient. For example, in Figure 5, dissolution results are shown for a proprietary BMS immediate-release product. When we tested these capsules, no significant difference $(<1 \%$ of dissolved) was observed for the dissolution profiles obtained with baseline correction at $350 \mathrm{~nm}$ versus correction over the range of $325-400 \mathrm{~nm}$.

Wavelength-dependent interference is generated by very small particles that form colloidal suspensions in the solution. Spectra measured from such solutions have an offset that usually increases at the lower wavelengths. Bynum et al ${ }^{28}$ utilized a second-derivative algorithm to correct wavelength-dependent scattering effects. The algorithm was only available on the Rainbow system. Recently this function has been added to the Opt-Diss system, but not to the IO system.

\section{Particulate Accumulation Effect}

Undissolved particles and excipients quite often pass through the detection windows of the fiber optic probes during the dissolution test. But they may also accumulate on the surface of the probe window. Accumulated particulates may block the UV light and generate erroneously high absorbance values and unrealistic dissolution data. This may be especially problematic when using shaft probes and rod probes. These two types of probes have a vertical optical path and a face-up mirror that are very likely to collect particulates. The arch probe, however, has a horizontal optical path. The probe tip cross-section is very small, and the optical surfaces are placed vertically, making them less likely to collect particulates during testing.

When we tested an extended-release formulation of a 20-mg tablet product, we observed different levels of particulate accumulation effects on different fiber optic systems. Figure 6 shows the dissolution profiles generated for this formulation using the arch probe/Opt-Diss system. These profiles were obtained using six probes and did not display any particulate accumulation effect. They matched the manual result reasonably well, even though the manual result was obtained for a different batch of the same formulation. When the rod probes on Rainbow and IO systems were used, the probe window collected a pile of pink particulate matter from the tablet excipients. The collected particulates generated absorbance artifacts and distorted dissolution profiles.

Based on our experiences, we believe the particulate accumulation on the rod probes depends on several factors. (1) The pharmaceutical formulations being tested: the effect is more severe for tablets that break down into large particles during dissolution testing. (2) Type of dissolution apparatus: the effect is more severe for USP Apparatus 1 than for Apparatus 2. (3) Agitation strength of the apparatus: the effect increases when the shaft rotation speed is decreased. (4) Test duration: the effect is more 


\section{In-Situ Dissolution Testing Using Different ... continued}

severe for extended release formulations than for immediate release formulations, and the longer the test duration, the greater the possibility for and extent of particulate accumulation.

A methodology to eliminate the particulate accumulation effect has been suggested by instrument vendors. A manifold installed on the dissolution bath may be used to raise the rod probes to just below the medium surface after each measurement occurs at the pre-determined time points. The probes are lowered to their proper USP sampling location only when measurement is imminent. It has been proposed that this mechanism should not only decrease the hydrodynamic effect from rod probes, but should also greatly decrease any particulate accumulation effect. We tested this methodology and moved the probes up and down every five minutes during the testing of the extended-release formulation. However, results from this experiment were not satisfactory, as the particulate accumulation was not eliminated. Further evaluation of this methodology is needed to arrive at a definitive solution.

\section{System Ruggedness and Flexibility}

Ruggedness and flexibility are also important factors in the application of this technology. As mentioned previously, the rod probes generally have larger dimensions, which can be a disadvantage, but that also makes them sturdy, easy to handle, and hard to break. The arch probes have much smaller dimensions, but their detection pathlength is determined by a small arch, which is relatively fragile and could easily become misaligned. Therefore, special care is needed when using the arch probes in dissolution testing.

An advantage of the rod probes is the ability to change pathlengths by using different probe tips. With changeable tips, both Delphian and C Tech rod probes can accommodate $0.2-, 0.5-, 1.0-$ and $2.0-\mathrm{cm}$ pathlengths, which is more economical and affords quick and easy changes. In contrast, the pathlength of shaft probes and arch probes is fixed at the time of manufacture, and therefore a different probe must be used when another pathlength is desired. The cost of a shaft or arch probe is much higher than that of a rod probe tip.

In addition, flexibility in setup, cleaning, and standardization are also factors to be considered when selecting a user-friendly system. For these operations, the shaft probe is difficult to use, but both rod probes and arch probes are relatively easy. A summary of these probe design performance factors is shown in Table 5.

\section{Conclusion}

UV fiber optics is a powerful tool for in-situ dissolution testing. We have generated data for a number of different drug products demonstrating equivalency of dissolution test results between fiber-optic and traditional UV detection. Current commercial fiber optic instrumentation exhibits great technological capabilities. Elimination of external sampling and filtration overcomes many of the limitations and problems associated with those steps, and reduces consumable-related expenses. Generation of test results is virtually instantaneous. Data can be acquired at a much higher frequency during early sampling time points, which could aid formulation development by yielding information about the mechanisms of initial drug release. Acquisition of data at more frequent time points might also enhance the ability to obtain a more discriminating test profile. Fiber optic detection can be applied efficiently for kinetic-type measurements within the dissolution vessels. However, there are still inherent limitations and challenges due to the spectroscopic characteristics and fiber optic probe designs of the different UV fiber optic dissolution systems. Learning about and understanding these characteristics and probe designs will help one to select a suitable instrument and to successfully conduct insitu dissolution testing for a variety of pharmaceutical solid dosage formulations.

\section{References:}

1. Josefson M., Johansson E., and Tortensson A., Analytical Chemistry (1988) 60, 2666-2671.

2. Brown C.W., Lin J., Applied Spectroscopy, (1993) 47, 615-618.

3. Chen C.S., Brown C.W., Pharmaceutical Research, (1994) 11 (7), 979-983.

4. Cho J.H., Gemperline P.J., Salt A., Walker D.S., Analytical Chemistry, (1995) 67, 2858-2863.

5. Cho J.H., Gemperline P.J.,Walker D.S., Applied Spectroscopy, (1995) 49 (12), 1841-1845.

6. Zhu B., Xing J.F., Chen J., Yaoxue Xuebao, (1994) 29 (5), 369-374.

7. Guo J., Chen J.,Yaowu Fenxi Zazhi, (1997) 17 (4), 228-231.

8. Gemperline P.J., Cho J.H., Baker, B., Batchelor B.,Walker D.S., Anal. Chem. Acta, (1997) 345 (1-3), 155-159.

9. Aldridge P.K., Melvin D.W., Williams B.A., Bratin K., Kostek L.J., Sekulic S.S., J. Pharmaceutical Science, (1995) 84 (8), 909-914.

10. Inman G.W., Martin W., Pharmaceutical Laboratory, (1998) 1 (1) 46.

11. Inman G.W., Wethington E., Baughman K. Horton M., Pharmaceutical Technology, (2001) 25 (10), 92-100.

12. Bynum K.C., Kraft E., Pharmaceutical Technology, (1999) 23 (10), 42-44.

13. Bynum K.C., Kraft E., Pocreva J., Ciurczak E.W., Palermo P., Dissolution technologies, (1999) 6 (4) 8-10.

14. Earnhardt J., Nir I.,Spectroscopy, (2000) 15 (2) 30-38. 
15. Nir I., Johnson B.D., Johansson J., Schatz C., Pharmaceutical Technology, (2001) 25 (5) 33-40.

16. Schatz C., Ulmschneider M., Altermatt R., Marrer S., Altorfer H., Dissolution Technologies, (2000) 7 (2) 6-13.

17. Schatz C., Ulmschneider M., Altermatt R., Marrer S., Altorfer H., Dissolution Technologies, (2000) 8 (2) 6-11.

18. Peng Y., Qu W., Sun Y., Johnson J., Shukla A., Pharmaceutical Formulation and Quality, (2003) 5 (4), 40-52.

19. Gray, V., American Pharmaceutical Review, (2003) 6 (2), 26-30.

20. The United States Pharmacopeial Convention, Inc., Note on USP Dissolution Calibrator Tablets, Rockville (2000).

21. The United States Pharmacopoeia, (2003), <711> Dissolution, 2156.

22. Palermo P., AAPS Dissolution Workshop, May 2001, at Arlington, VA.

23. Schatz C., Ulmschneider M., Altermatt R., Marrer S., Dissolution Technology, (2000) 7 (1), 20-21.

24. Schatz C., Ulmschneider M., Altermatt R., Marrer S., Altorfer H., Spectroscopy Europe, (2000) 12 (6) 12-18.

25. Lu X., Lo L., Lozano R., Ross-Montgomery E., Shah P., Presentation at Eastern Analytical Symposium, (2002)

26. Schatz C., Ulmschneider M., Altermatt R., Marrer S., Dissolution technologies, (2000) 7 (4), 8-17.

27. Operational Qualification Procedure: Spectroscopic Measurement Functions for Opt-Diss Dissolution Testing System, Leap Technologies Inc., (2002)

28. Bynum K., Roinestad K., Kassis A., Pocreva J., Gehrlein L., Cheng F., Palermo P., Dissolution Technologies, (2001) 8 (4), 13-22. 